\title{
Serial echocardiographic evaluation of the Perimount Magna Ease prosthesis
}

\author{
Benedikt Mayr ${ }^{1,2 \#} \wedge$, Melchior Burri ${ }^{1,2 \#}$, Keti Vitanova ${ }^{1,2}$, Anatol Prinzing ${ }^{1,2}$, Gertrud Goppel $^{1,2}$, \\ Markus Krane ${ }^{1,2,3}$, Rüdiger Lange ${ }^{1,2,3}$, Ralf Günzinger ${ }^{1,2}$ \\ ${ }^{1}$ Department of Cardiovascular Surgery, German Heart Center Munich, Technische Universität München, Munich, Germany; ${ }^{2}$ Insure (Institute for \\ Translational Cardiac Surgery), Department of Cardiovascular Surgery, German Heart Center Munich, Technische Universität München, Munich, \\ Germany; ${ }^{3}$ DZHK (German Center for Cardiovascular Research)—partner site Munich Heart Alliance, Munich, Germany \\ Contributions: (I) Conception and design: B Mayr, R Günzinger, R Lange; (II) Administrative support: R Günzinger, M Krane, R Lange; (III) \\ Provision of study materials: B Mayr, G Goppel; (IV) Collection and assembly of data: B Mayr, M Burri; (V) Data analysis and interpretation: B \\ Mayr, R Günzinger, M Burri, K Vitanova; (VI) Manuscript writing: All authors; (VII) Final approval of manuscript: All authors. \\ "These authors contributed equally to this work. \\ Correspondence to: Benedikt Mayr, MD. Department of Cardiovascular Surgery, German Heart Center Munich, Lazarettstraße 36, 80636 Munich, \\ Germany. Email: mayrb@dhm.mhn.de.
}

Background: The Carpentier-Edwards Perimount Magna Ease prosthesis (PME) represents the latest generation of stented bioprostheses used for surgical aortic valve replacement (SAVR). The aim of our study was to evaluate the long-term clinical outcome and hemodynamic performance of the prosthesis with a focus on the incidence and course of structural valve deterioration (SVD) by serial echocardiographic examinations.

Methods: SAVR with the PME was performed in 58 consecutive patients between 2007 and 2008. Transthoracic echocardiography was performed preoperatively, at discharge and annually during a 10-year follow-up at the German Heart Center Munich.

Results: Mean age at surgery was $62 \pm 14$ years. At discharge $(\mathrm{n}=57)$, the overall mean pressure gradient (MPG) and effective orifice area (EOA) were $15.8 \pm 4.1 \mathrm{mmHg}$ and $1.8 \pm 0.4 \mathrm{~cm}^{2}$, respectively. Moderate patient-prosthesis mismatch (PPM) was present in 18 patients $(32 \%)$ and severe PPM in 6 patients $(11 \%)$ at discharge. Ten years following SAVR ( $\mathrm{n}=33$ ), the overall MPG was $16.6 \pm 7.3 \mathrm{mmHg}$ and EOA was $1.3 \pm 0.4 \mathrm{~cm}^{2}$. Thirty-day and late mortality was $2 \%(\mathrm{n}=1)$ and $21 \%(\mathrm{n}=12)$, respectively. Survival at 1 , 5 , and 10 years was $94.7 \% \pm 3.3 \%, 91.1 \% \pm 4.1 \%$, and $77.3 \% \pm 5.9 \%$, respectively. Freedom from reoperation at 10 years was $88.8 \% \pm 4.7 \%$. Ten years after PME implantation the cumulative incidence of any SVD, severe SVD, and bioprosthetic valve failure (BVF) was $25 \% \pm 6 \%, 14 \% \pm 5 \%$, and $16 \% \pm 5 \%$, respectively.

Conclusions: The PME shows an excellent hemodynamic performance over the course of 10 years with development of clinically relevant SVD as late as 6 years post implant, and a 10-year incidence of severe SVD of $14 \%$.

Keywords: Aortic valve stenosis; aortic valve replacement; structural valve deterioration (SVD)

Submitted Mar 18, 2021. Accepted for publication May 14, 2021.

doi: $10.21037 /$ jtd-21-481

View this article at: https://dx.doi.org/10.21037/jtd-21-481

\footnotetext{
^ ORCID: 0000-0002-1164-0746.
} 


\section{Introduction}

Bioprosthetic heart valves (BHV) are predominantly used for surgical aortic valve replacement (SAVR) $(1,2)$. This trend has been intensified by the introduction of transcatheter aortic valve replacement (TAVR) providing an alternative to redo surgery in patients with failed bioprostheses (3). BHV are of limited durability due to the occurrence of structural valve deterioration (SVD). In the past, SVD has often been described by the rate of freedom from reoperation leading to an underestimation of the incidence of SVD $(4,5)$. Consequently, SVD should be assessed by clinically detectable measures using echocardiographic criteria other than the need for reoperation for a failing bioprosthesis (6). For the proper assessment of SVD, a close and complete serial echocardiographic follow-up is mandatory (6-8). Currently, only data on early and mid-term hemodynamic and clinical performance of the Carpentier-Edwards Perimount Magna Ease prosthesis (PME) (Edwards Lifesciences, Irvine, CA, USA) are available $(9,10)$. Specific characteristics of this stented bovine pericardial aortic valve are its cobaltchromium alloy stent, the low profile, the low stent base, and sleek commissure posts. The aim of our investigation was to determine the long-term performance of the PME with special attention to the incidence and course of SVD.

We present the following article in accordance with the STROBE reporting checklist (available at https://dx.doi. org/10.21037/jtd-21-481).

\section{Methods}

\section{Study design and study population}

The study was conducted in accordance with the Declaration of Helsinki (as revised in 2013). This study was approved by the Institutional Review Board of the Technical University Munich (ID: 174/19 S-SR, Date: 21.05.2019) and the need for informed patient consent was waived due to the retrospective nature of the study. Patients undergoing SAVR with the PME between October 2007 and September 2008 ( $\mathrm{n}=58)$ with annual echocardiographic follow-up were identified retrospectively in our institutional database. Patients with concomitant other valve procedures were excluded. Previous cardiac surgery had not been performed in any patient. Reasons for SAVR were stenosis in 29 patients (50\%), combined disease in 24 patients (41\%), and regurgitation in 5 patients (9\%). Baseline characteristics are shown in Table 1.

\section{Implantation technique}

SAVR was performed through median sternotomy in 21 patients (36\%) or upper hemisternotomy in 37 patients (64\%). The most frequent concomitant procedure was coronary artery bypass grafting in 19 patients (33\%). Cardiopulmonary bypass was established in the usual manner and cold crystalloid cardioplegia was administrated antegrade. Selection of the adequate prosthesis's size was based on the manufacturer's aortic annulus sizers. Supraannular implantation of the prosthetic valve was performed with pledgeted interrupted non-everting mattress sutures. Procedural data are shown in Table 2.

\section{Anticoagulation management}

After operative treatment, all patients were anticoagulated with phenprocoumon for 3 months. Anticoagulation was continued if there were other indications for permanent use.

\section{Follow-up}

After implantation of the PME, clinical and echocardiographic follow-up was performed annually for up to 10 years at our center according to a standardized protocol by the same group of cardiologists. Mean follow-up time was 8.3 \pm 3 years. Six patients (10\%) discontinued follow-up at 0,4 , $6,8,8$, and 9 years.

\section{Transthoracic echocardiography}

Transthoracic echocardiography was performed preoperatively, at discharge, and annually during followup period. Measurement of left ventricular dimensions was performed using the M-mode. Left ventricular ejection fraction was evaluated in biplanar images using the Simpson method. Left ventricular hypertrophy was calculated by the formula for left ventricular mass according to Devereux et al. (11). Left ventricular mass was indexed to the body surface area. Mean pressure gradient (MPG) as well as transor paraprosthetic regurgitation were determined using Doppler echocardiography. Effective orifice area (EOA) was determined using the continuity equation. Patientprosthesis mismatch (PPM) was calculated using the indexed effective orifice area at discharge (iEOA) (12). PPM was graded as none $\left(\mathrm{iEOA}>0.85 \mathrm{~cm}^{2} / \mathrm{m}^{2}\right)$, moderate $(\mathrm{iEOA}=0.85$ $\left.-0.65 \mathrm{~cm}^{2} / \mathrm{m}^{2}\right)$, or severe (iEOA $\left.<0.65 \mathrm{~cm}^{2} / \mathrm{m}^{2}\right)(12)$. Evaluation of non-structural valve dysfunction (NSVD), 
Table 1 Baseline characteristics of the study population

\begin{tabular}{|c|c|}
\hline Characteristic & Outcome \\
\hline \multicolumn{2}{|l|}{ Demographics } \\
\hline Patients & 58 [100] \\
\hline Age (years) & $66.1 \pm 9.4$ \\
\hline Gender, female & $16[28]$ \\
\hline BMI $\left(\mathrm{kg} / \mathrm{m}^{2}\right)$ & $26.5 \pm 3.8$ \\
\hline NYHA class IV & 0 \\
\hline NYHA class III & 13 [23] \\
\hline NYHA class II & $35[60]$ \\
\hline NYHA class I & 10 [17] \\
\hline \multicolumn{2}{|l|}{ Comorbidities } \\
\hline Arterial hypertension & $38[66]$ \\
\hline Smoking (ex and current) & $20[34]$ \\
\hline Diabetes mellitus & $9[16]$ \\
\hline Pulmonary disease & $3[5]$ \\
\hline Renal disease & $1[2]$ \\
\hline Liver disease & $4[7]$ \\
\hline Coronary artery disease & $32[55]$ \\
\hline Ascending aortic aneurysm & $8[13]$ \\
\hline Peripheral vascular disease & 5 [9] \\
\hline Previous stroke & 0 \\
\hline Atrial fibrillation & 2 [3] \\
\hline \multicolumn{2}{|l|}{ Valve pathology } \\
\hline Stenosis & $29[50]$ \\
\hline Regurgitation & 5 [9] \\
\hline Mixed disease & $24[41]$ \\
\hline Bicuspid valves & $15[26]$ \\
\hline Endocarditis & 0 \\
\hline Rheumatic fever & $1[2]$ \\
\hline Mean LVEF (\%) & $63 \pm 11$ \\
\hline EuroSCORE II (\%) & $1.2 \pm 0.7$ \\
\hline
\end{tabular}

Values are expressed as $\mathrm{n}[\%]$ or mean \pm standard deviation. BMI, body mass index; LVEF, left ventricular ejection fraction; NYHA, New York Heart Association.
Table 2 Peri- and intraoperative details

\begin{tabular}{lc}
\hline Variable & Outcome \\
\hline Median sternotomy & $21[36]$ \\
Partial upper sternotomy & $37[64]$ \\
Prosthesis size (mm) & \\
19 & $2[4]$ \\
21 & $6[11]$ \\
23 & $17[30]$ \\
25 & $27[47]$ \\
27 & $4[7]$ \\
29 & $1[2]$ \\
Concomitant procedures & \\
CABG & $19[33]$ \\
Reduction ascending aortoplasty & $6[10]$ \\
Supracoronary ascending aortic replacement & $2[3]$ \\
Aortic cross-clamp time (minutes) & $65.8 \pm 17$ \\
Cardiopulmonary bypass time (minutes) & $91.1 \pm 21$ \\
Ventilation time (hours) & $8[3 \mathrm{~h}-13 \mathrm{~d}]$ \\
ICU stay (days) & $1.5[1-13]$ \\
Hospital stay (days) & $7[4-29]$ \\
\hline Operative mortality (\%) & $1[2]$ \\
\hline
\end{tabular}

Values are expressed as $\mathrm{n}$ [\%] or median [interquartile range]. $\mathrm{CABG}$, coronary artery bypass grafting; ICU, intensive care unit; $\mathrm{mm}$, milimeter.

SVD, and bioprosthetic valve failure (BVF) was done according to the standardized definitions of structural deterioration and valve failure by Capodanno et al. (6) (Figure 1). NSVD is characterized by a morphologic not altered prosthetic valve but with impaired function due to leaflet thrombosis, endocarditis, intra- or para-prosthetic regurgitation or PPM. SVD is characterized by intrinsic permanent changes of the prosthetic valve either without hemodynamic impairment $($ morphologic $=$ stage 1$)$ or with hemodynamic impairment $($ moderate $=$ stage 2 ; severe = stage 3 ) (Figure 1). If the patient's clinical condition is 


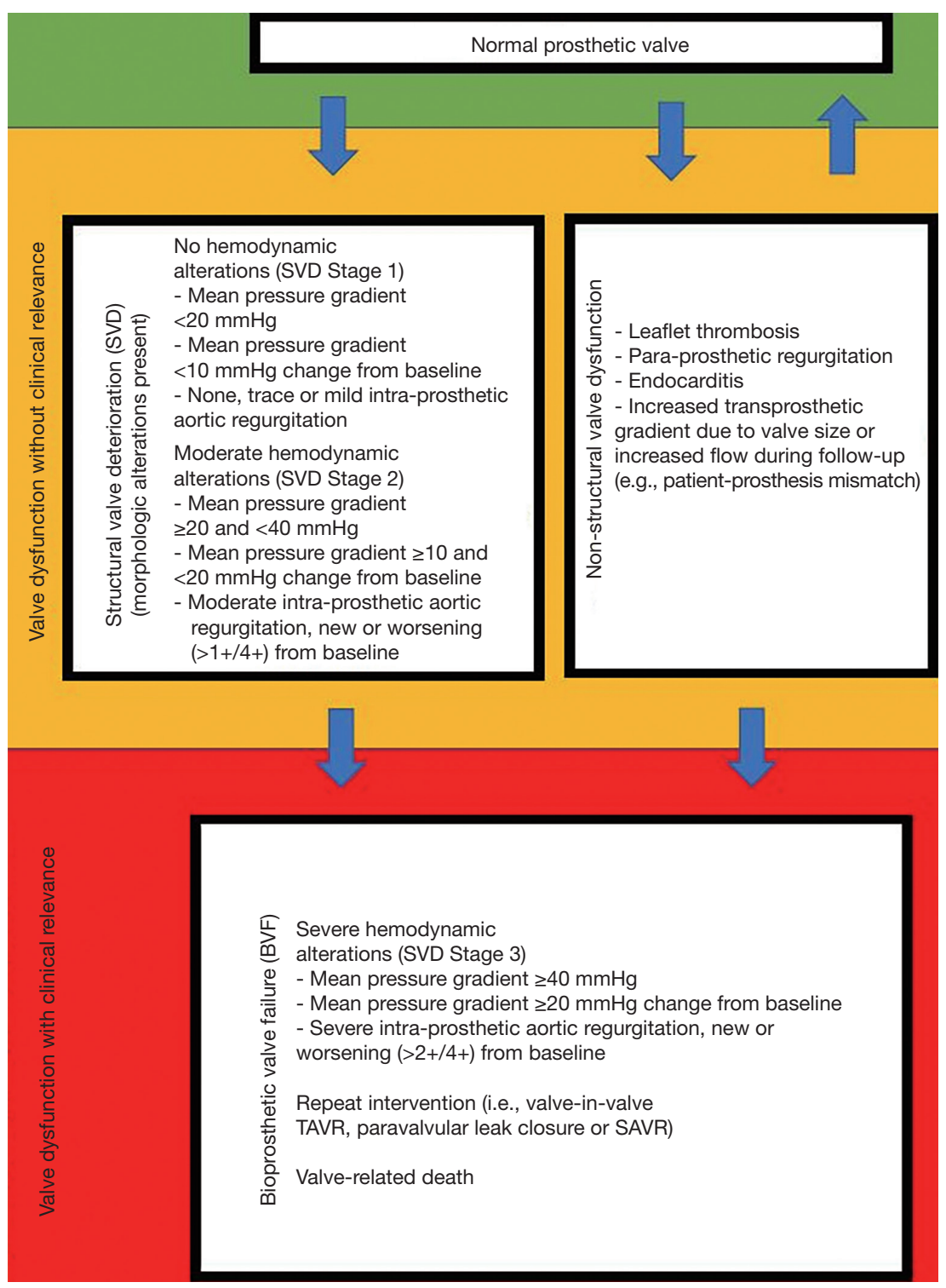

Figure 1 Classification of non-structural valve dysfunction, structural valve deterioration, and bioprosthetic valve failure according to Capodanno et al. (6). SAVR, surgical aortic valve replacement; SVD, structural valve deterioration; TAVR, transcatheter aortic valve replacement.

impaired by SVD Stage 3 or NSVD resulting in valverelated death or repeat intervention (valve-in-valve TAVR, paravalvular leak closure or SAVR) the criteria for BVF are met (Figure 1). The timepoint of NSVD, SVD, and BVF was determined by graphing all echocardiographic data for each patient individually (supplementary material, available online: https://cdn.amegroups.cn/static/public/jtd-21-481Supplementary.pdf).

\section{Statistical analysis}

Data were analyzed using R (version 3.5.2; R Foundation for Statistical Computing, Vienna, Austria). Descriptive statistics are described as frequencies and percentages for categorical variables. Continuous variables are reported as mean \pm standard deviation if normally distributed and as median with 1 st and 3 rd quartiles $(\mathrm{Q} 1 ; \mathrm{Q} 3)$ if non normally 

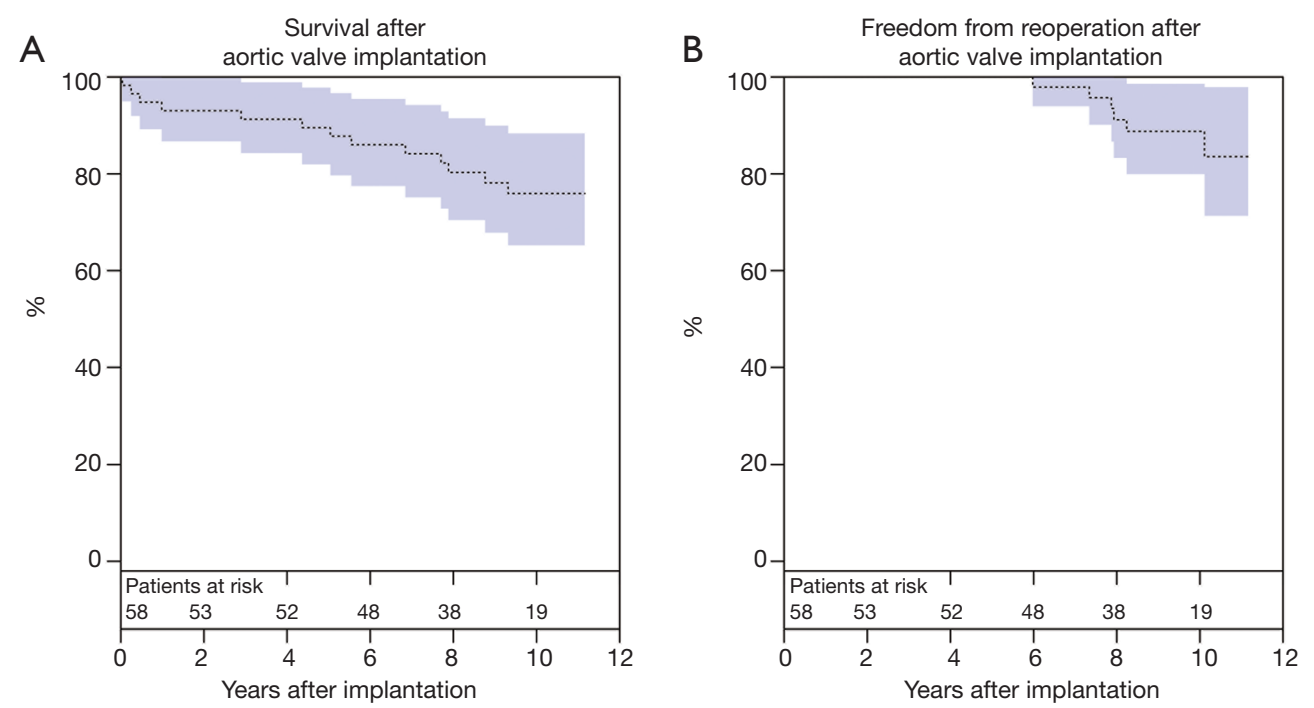

Figure 2 Long-term outcome after aortic valve implantation of the PME. (A) Survival after aortic valve implantation. (B) Freedom from reoperation after aortic valve implantation. PME, Carpentier-Edwards Perimount Magna Ease prosthesis.

distributed. Confidence interval (CI) is reported as $95 \%$ CI. Kaplan-Meier analysis was applied to calculate estimated survival and freedom from reoperation. SVD, NSVD, and BVF were analyzed with a competitive risk analysis calculating the cumulative incidence. Statistical significance was set at $\mathrm{P}<0.05$.

\section{Results}

\section{In-bospital adverse events}

One patient died from multiple organ failure on postoperative day 13 after SAVR. Rethoracotomy due to bleeding was performed in three patients $(5 \%)$. Other adverse events included requirement for dialysis in one patient (2\%), postoperative transient ischemic attack (TIA) in one patient, and need for postoperative pacemaker implantation in two patients (3\%).

\section{Adverse events during follow-up}

Late mortality was $21 \%(\mathrm{n}=12)$ during follow-up. Survival at 1,5 , and 10 years was $94.7 \% \pm 3.4 \%, 91.1 \% \pm 4.1 \%$, and $77.3 \% \pm 5.9 \%$, respectively (Figure $2 A$ ). Causes of noncardiac death $(n=9)$ were: cancer $(n=4)$, multiple organ failure $(n=3)$, upper gastrointestinal bleeding $(n=1)$, and pneumonia $(n=1)$. Cause of cardiac death $(n=3)$ were: prosthetic valve endocarditis $(n=1)$, heart failure $(n=1)$, and unknown $(\mathrm{n}=1)$. Pacemaker implantation after hospital discharge was required in five (9\%) patients due to: highgrade atrioventricular block $(n=4)$ and bradyarrhythmia absoluta $(\mathrm{n}=1)$. Major bleeding occurred in three patients $(5 \%)$ and minor bleeding in six patients $(10 \%)$. Stroke was observed in two patients (3\%) and TIA in seven patients $(12 \%)$. Myocardial infarction occurred in three patients $(5 \%)$. Freedom from reoperation at 10 years was $88.8 \% \pm 4.7 \%$ (Figure $2 B$ ). Six patients $(10 \%)$ underwent reoperation at $6,7,8,8,8$ and 10 years due to BVF. EuroSCORE II at time of reintervention was $4.2 \% \pm 1.5 \%$. In all six patients, a valve-in-valve TAVR was successfully performed.

\section{Hemodynamic results}

After SAVR, the MPG significantly decreased $(59 \pm 17 v s$. $16 \pm 4 \mathrm{mmHg}, \mathrm{P}<0.001)$ and the EOA significantly increased $\left(0.8 \pm 0.22\right.$ vs. $\left.1.75 \pm 0.42 \mathrm{~cm}^{2}, \mathrm{P}<0.001\right)$. MPGs and EOAs at discharge and at 10-year follow-up are shown in Table 3. At discharge $(\mathrm{n}=57)$, moderate PPM was seen in 18 patients $(32 \%)$ and severe PPM in 6 patients $(11 \%)$. No intraor para-prosthetic regurgitation was seen at any time. A detailed description of survival, BVF, SVD and NSVD depending on the presence of PPM at discharge is attached in the Supplementary Appendix. A significant regression of the indexed left ventricular mass was observed within the first year after implantation $\left(139 \pm 41\right.$ vs. $109 \pm 25 \mathrm{gram} / \mathrm{m}^{2}$, $\mathrm{P}<0.001)$. EOAs, MPGs, and indexed left ventricular mass 
Table 3 Valve hemodynamics at discharge and 10-year follow-up

\begin{tabular}{|c|c|c|c|c|c|c|c|}
\hline Valve sizes & All sizes & $19 \mathrm{~mm}$ & $21 \mathrm{~mm}$ & $23 \mathrm{~mm}$ & $25 \mathrm{~mm}$ & $27 \mathrm{~mm}$ & $29 \mathrm{~mm}$ \\
\hline $\mathrm{n}$ & 57 & 2 & 6 & 17 & 27 & 4 & 1 \\
\hline MPG (mmHg) & $15.8 \pm 4.1$ & $17.73 \pm 0.18$ & $20.27 \pm 5.22$ & $16.03 \pm 3.53$ & $15.17 \pm 3.88$ & $12.65 \pm 1.08$ & 11.2 \\
\hline $\mathrm{EOA}\left(\mathrm{cm}^{2}\right)$ & $1.8 \pm 0.4$ & $1.08 \pm 0.04$ & $1.37 \pm 0.38$ & $1.76 \pm 0.32$ & $1.81 \pm 0.40$ & $2.11 \pm 0.51$ & 2.15 \\
\hline $\mathrm{n}$ & 33 & 1 & 2 & 10 & 17 & 2 & 1 \\
\hline MPG $(\mathrm{mmHg})$ & $16.6 \pm 7.3$ & 22 & $29.50 \pm 20.51$ & $16.80 \pm 6.27$ & $15.12 \pm 5.06$ & $10.00 \pm 2.83$ & 20 \\
\hline $\mathrm{EOA}\left(\mathrm{cm}^{2}\right)$ & $1.3 \pm 0.4$ & 0.83 & $0.98 \pm 0.25$ & $1.29 \pm 0.45$ & $1.37 \pm 0.30$ & 1.54 & 1.3 \\
\hline
\end{tabular}

Values are expressed as $\mathrm{n}(\%)$ or mean \pm standard deviation. $\mathrm{cm}$, centimeter; EOA, effective orifice area; mm, millimeter; MPG, mean pressure gradient.
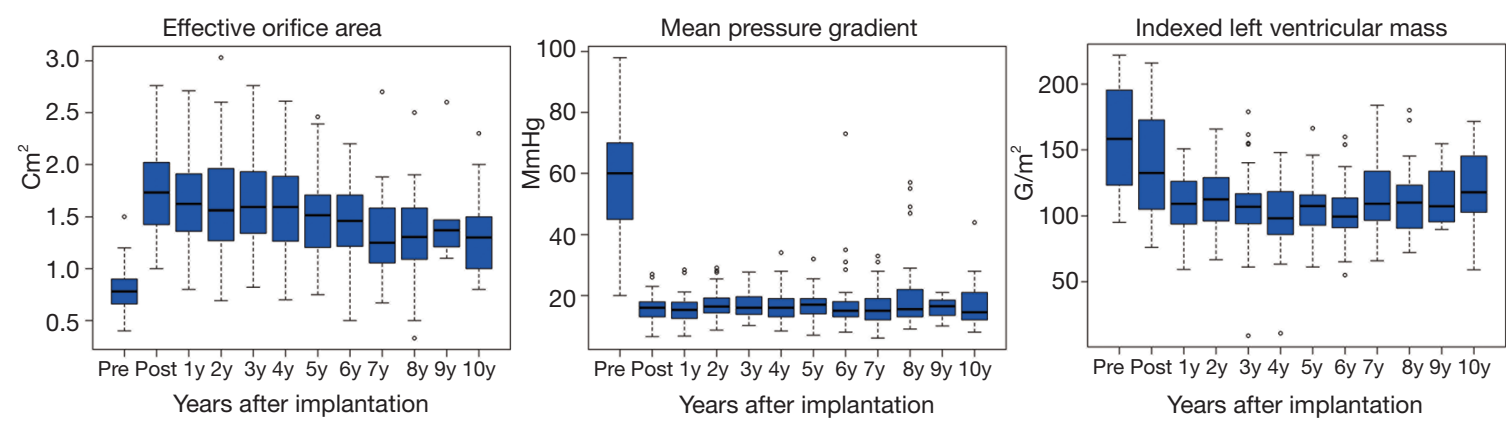

Figure 3 Hemodynamic parameters after PME implantation during the 10-year follow-up. (A) Effective orifice area. (B) Mean pressure gradient. (C) Indexed left ventricular mass. PME, Carpentier-Edwards Perimount Magna Ease prosthesis.

throughout the entire follow-up period are shown in Figure 3.

\section{Valve durability}

The cumulative incidence of any SVD (stage 1, stage 2, and stage 3 ) during the 10 -year follow-up was $25 \% \pm 6 \%$ (Figure $4 A$ ). Freedom from any SVD at 5 and 10 years was $100 \% \pm 0 \%$ and $68.9 \% \pm 7.3 \%$, respectively. Earliest morphologic alterations of the PME occurred 5 years after implantation, the average time was $8.2 \% \pm 1.9 \%$ years. The cumulative incidence of SVD with at least moderate hemodynamic alterations ( $\geq$ stage 2 ) was $20 \% \pm 6 \%$ and the cumulative incidence with severe hemodynamic alterations (stage 3 ) was $14 \% \pm 5 \%$. Freedom from SVD stage 3 at 5 and 10 years was $100 \% \pm 0 \%$ and $82.8 \% \pm 6.1 \%$, respectively. The cumulative incidence of BVF was $16 \% \pm 5 \%$ (14\%: SVD stage $3 ; 2 \%$ : death due to prosthetic valve endocarditis) (Figure 4B). During follow-up, NSVD occurred in
30 patients (52\%) of whom 29 patients presented with an increased MPG higher than $20 \mathrm{mmHg}$ but no morphologic alterations of the prosthesis, and 1 patient with prosthetic valve endocarditis. In $10 \%$ (3/30) NSVD progressed to SVD stage 2 and in 16\% (5/30) NSVD changed to SVD stage 3 . In $37 \%$ (11/30) NSVD was only temporary and in $37 \%(11 / 30)$ NSVD was diagnosed at the last followup (Figure 5). Highest temporary increase in MPG was $24 \mathrm{mmHg}$ in a patient with a $21 \mathrm{~mm}$ PME (iEOA: $0.94 \mathrm{~cm}^{2}$ ) 3 years after implantation. In the 5- and 8-year follow-up, the patient had an MPG of 18 and $15 \mathrm{mmHg}$, respectively.

\section{Discussion}

Our study and follow-up was conducted according to the 2017 ESC and EACTS guidelines, with annual echocardiography up to 10 years following the implantation of the Carpentier-Edwards Perimount Magna Ease 

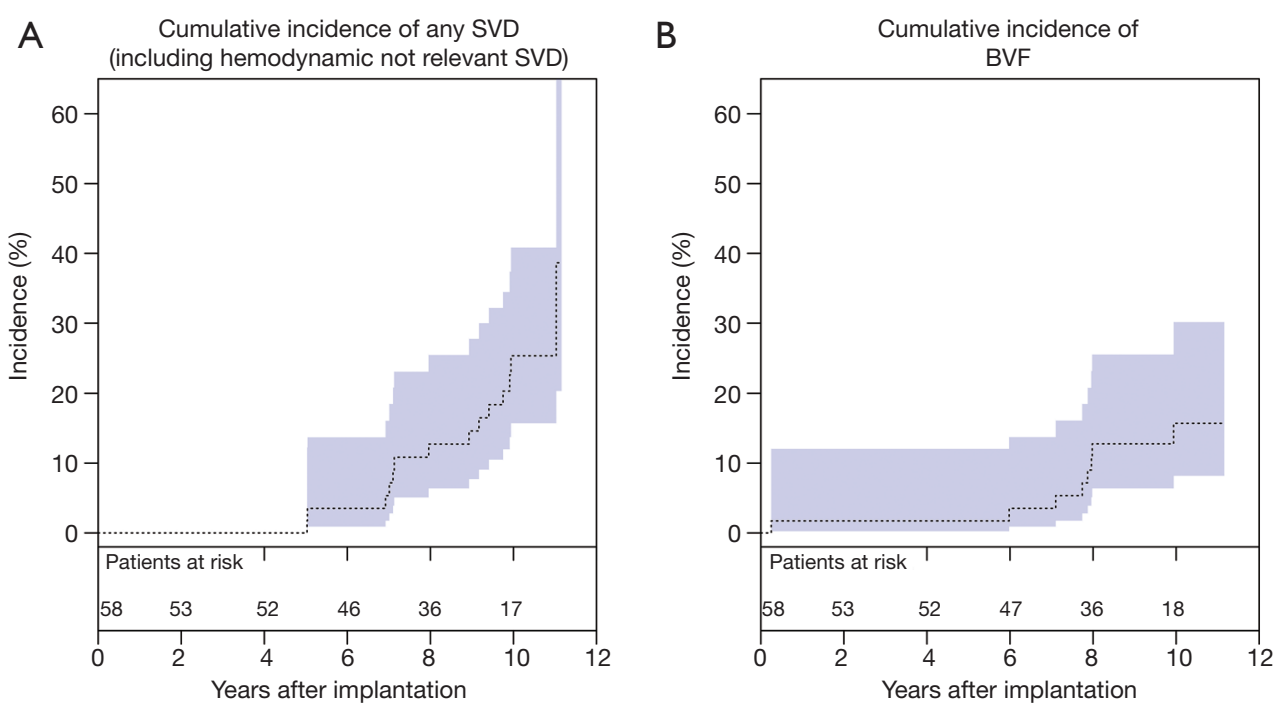

Figure 4 Cumulative incidence of PME deterioration. (A) Cumulative incidence of any SVD. (B) Cumulative incidence of BVF. PME, Carpentier-Edwards Perimount Magna Ease prosthesis; SVD, structural valve deterioration; BVF, bioprosthetic valve failure.

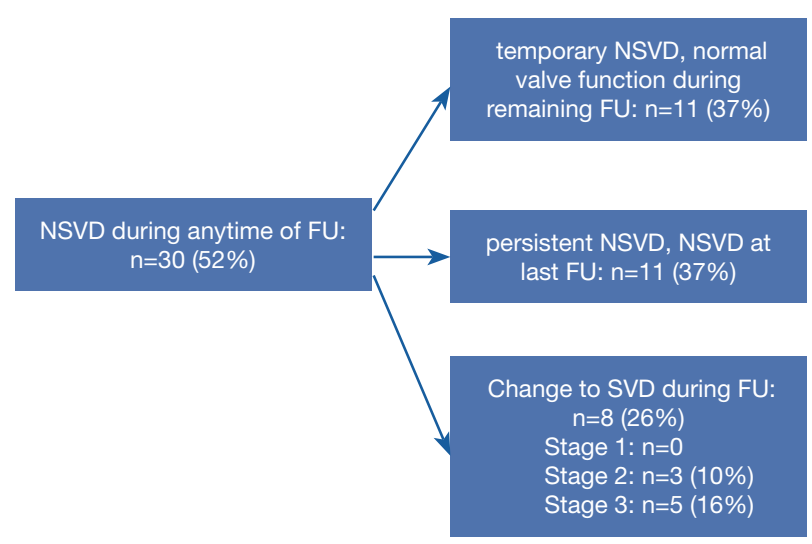

Figure 5 Course of non-structural valve dysfunction. FU, follow-up; NSVD, non-structural valve dysfunction; SVD, structural valve deterioration.

prosthesis to investigate the incidence and course of SVD (6). A recent review about the durability of bioprosthetic aortic valves published by Salaun et al. emphasized that the rate of bioprosthetic valve durability is often reported as valve reoperation and not as the rate of SVD (8). A certain proportion of patients with SVD might not undergo reoperation due to undetected SVD and SVD in frail and elderly patients with major comorbidities presenting a high risk for surgical reoperation (8). Thus, the true incidence and impact of SVD are underestimated and BHV durability is overestimated (8). Consequently, SVD should be defined on the basis of new onset or worsening of morphological and hemodynamic alterations of BHV by serial Doppler echocardiography (6). In the present investigation, the PME showed an excellent hemodynamic performance with low MPGs and stable EOAs during a 10-year follow-up. Ten years after SAVR with the PME freedom from reoperation was $89 \%$ with an incidence of SVD stage 3 and BVF of $14 \%$ and $16 \%$, respectively. The earliest morphologic alterations in echocardiography occurred 5 years after implantation. However, these alterations were not clinically relevant. The first clinically relevant SVD occurred 6 years after implantation. Therefore, it is mandatory that studies focusing on SVD after bioprosthetic aortic valve replacement have a mean follow-up of at least 5 years and serial echocardiographic evaluation. Moreover, annual echocardiographic follow-up by the referring cardiologists is mandatory for every patient having a BHV, especially after the fifth year of implantation. Currently, only reports on the mid-term hemodynamic performance of the PME are available and show an actuarial freedom from SVD at 5 years of $99 \%$ (10). This report is in line with our cumulative incidence of any SVD of $0 \%$ at 5 years. David et al. reported excellent durability of the Hancock II prosthesis (porcine, Medtronic, Minneapolis, MN, USA) in 1.134 patients with an incidence of SVD stage 3 of $2.4 \%$ at 10-year follow-up (13). However, David et al. followed their patients only every second year by questionnaires without regular echocardiographic examination of the implanted 
valve and might have missed the first signs of SVD (13). In the retrospective study by Yankah et al., a Sorin Mitroflow (bovine pericardial, Sorin Group Canada Inc. Mitroflow Division, BC, Canada) prosthetic valve implantation was performed in 1.513 patients and freedom from SVD at 10 years post-implant was $90 \%$ (14). However, in the investigation by Yankah et al., only $86 \%$ of the patients underwent postoperative annual echocardiography, which was performed at different institutions. Another very common stented bioprosthetic valve is the CarpentierEdwards Perimount prosthesis (bovine pericardial) with a reported incidence of SVD $\geq$ stage 2 of $5.8 \%$ at 10 years after SAVR (15). In the study by Bourguignon et al., 2.659 patients were followed with an echocardiogram at the time of follow-up (15). In our series, the incidence of SVD $\geq$ stage 2 at 10 -year follow-up was more frequent due to the lower mean age (by 5 years) of our study population and due to our continuous echocardiographic follow-up. New generation heart valves made with tissue designed to reduce calcification, such as the RESILIA (bovine pericardial, Edwards Lifesciences, Irvine, CA, USA) prosthesis, showed no SVD up to 5 years of implantation $(16,17)$. When comparing this valve to the PME, equal rates of SVD 5 years post implantation were seen, and the potential longterm advantage of the RESILIA prosthesis still needs to be proven.

SVD can now be managed with reasonable risks using valve-in-valve TAVR. However, the long-term durability of valve-in-valve TAVR remains largely unknown. Thus, the risk of open reoperation with known long-term durability of surgical bioprostheses still needs to be contemplated against valve-in-valve TAVR. In young patients and patients with small aortic annulus who are at risk for future reintervention due to SVD, aortic root enlargement in combination with SAVR facilitating the implantation of a larger prosthetic valve should be kept in mind. In the meta-analysis by $\mathrm{Yu}$ et al. aortic root enlargement was found to be a safe adjunct to SAVR resulting in larger implanted prosthetic valves and less PPM possibly improving future valve-in-valve TAVR outcomes (18). They could also show that addition of aortic root enlargement to SAVR does not increase early adverse events such as myocardial infarction, permanent pacemaker implantation, reoperation for bleeding, or stroke. However, aortic root enlargement increases the extent of the operation resulting in increased aortic cross-clamp time which was associated with increased late mortality (19). In the present study, none of the patients received aortic root enlargement. Moderate and severe PPM was seen in
$32 \%$ and $11 \%$, respectively. This is similar to rates of PPM reported elsewhere: In the retrospective investigation by Anselmi et al., moderate and severe PPM is described to occur in $30 \%$ and $7 \%$, respectively, which is comparable to the data in our study (10). Deutsch and colleagues reported comparable numbers of moderate and severe PPM (24\% and 4\%) with the supraannular St Jude Medical Trifecta prosthesis (bovine pericardial, St Jude Medical, Inc., St Paul, MN, USA) (20).

In the present investigation overall survival at 10 years was $77 \%$. This compares favorably to Anselmi et al. who report a survival rate of $74 \%$ seven years after PME implantation (10). Other reported survival rates 10 years after SAVR with various stented bioprosthetic valves range from $32 \%$ to $95 \%$, depending on the age of the patient population as well as the rate of concomitant procedures $(4,13-15)$. In the present investigation, three patients (5\%) died due to cardiac causes. During the 10-year followup, a MPG greater than $20 \mathrm{mmHg}$ without morphologic alterations of the PME was detected in 29 patients. In $37 \%$, the increase in MPG resolved in the following echocardiographic check-ups. In 33\%, the MPG remained increased but did not progress. Only 26\% progressed to SVD. This shows that the MPG can fluctuate during serial echocardiographic follow-up. Reasons for this might include variations of the patient's volume status and heart rate and inter- and intra-observer variability. Another reason for persistent increased MPG might be caused by a PPM. Also, recent findings have shown that microthrombosis of prosthetic valve leaflets detected during four-dimensional computed tomography might be a cause of temporary elevated MPGs (21). However, this does not consequently imply the development of SVD. These facts highlight the need for annual echocardiographic follow-up.

The present single-center study was limited by its nonrandomized and retrospective design. Furthermore, the study was limited by the relatively small number of patients and the available serial echocardiographic evaluation over the course of 10 years. Standardized follow-up protocol was performed in the initial patients, in whom a PME was implanted. Due to the small size of the groups, a statistical comparison of patients with none, moderate and severe PPM was not feasible and regression analysis for risk factors for SVD was not performed.

In conclusion, the PME shows an excellent performance over the course of 10 years after SAVR, with a 10 -year survival and freedom from reoperation rate of $77 \%$ and $89 \%$, respectively. Cumulative incidence of SVD stage 3 
at 10 years after PME implantation was $14 \%$. Worsening of NSVD with permanent morphologic alterations of the prosthetic valve was seen in $26 \%$ of patients. The first clinically relevant SVD occurred 6 years after implantation of the PME. Serial annual echocardiographic evaluations of BHVs are mandatory to detect morphologic alterations of the prosthesis and to follow the course of SVD.

\section{Acknowledgments}

The authors wish to thank BioMed Proofreading LLC for the editing of this manuscript by native English-speaking experts. Data obtained from the study: "Carpentier-Edwards PERIMOUNT Magna Ease Pericardial Bioprosthesis, Model 3300 TFX" (NCT01171625), funded by Edwards Lifesciences (Irvine, CA, USA), were used for this study.

Funding: None.

\section{Footnote}

Reporting Checklist: The authors have completed the STROBE reporting checklist. Available at https://dx.doi. org/10.21037/jtd-21-481

Data Sharing Statement: Available at https://dx.doi. org/10.21037/jtd-21-481

Peer Review File: Available at https://dx.doi.org/10.21037/ jtd-21-481

Conflicts of Interest: All authors have completed the ICMJE uniform disclosure form (available at https://dx.doi. org/10.21037/jtd-21-481). Dr. KV received speaker honoraria from Medtronic. Prof. MK received speaker honoraria from Medtronic and JOMDD. Prof MK is part of the advisory board of JOMDD. Prof. RL received grants from Boston Scientific and High Life. Prof. RL received speaker honoraria from Medtronic and he is part of the advisory board of Medtronic. The authors have no other conflicts of interest to declare.

Ethical Statement: The authors are accountable for all aspects of the work in ensuring that questions related to the accuracy or integrity of any part of the work are appropriately investigated and resolved. The study was conducted in accordance with the Declaration of Helsinki (as revised in 2013). This study was approved by the Institutional Review Board of the Technical University
Munich (ID: 174/19 S-SR, Date: 21.05.2019) and the need for informed patient consent was waived due to the retrospective nature of the study.

Open Access Statement: This is an Open Access article distributed in accordance with the Creative Commons Attribution-NonCommercial-NoDerivs 4.0 International License (CC BY-NC-ND 4.0), which permits the noncommercial replication and distribution of the article with the strict proviso that no changes or edits are made and the original work is properly cited (including links to both the formal publication through the relevant DOI and the license). See: https://creativecommons.org/licenses/by-nc-nd/4.0/.

\section{References}

1. Beckmann A, Meyer R, Lewandowski J, et al. German Heart Surgery Report 2018: The Annual Updated Registry of the German Society for Thoracic and Cardiovascular Surgery. Thorac Cardiovasc Surg 2019;67:331-44.

2. Isaacs AJ, Shuhaiber J, Salemi A, et al. National trends in utilization and in-hospital outcomes of mechanical versus bioprosthetic aortic valve replacements. J Thorac Cardiovasc Surg 2015;149:1262.

3. Baumgartner H, Falk V, Bax JJ, et al. ESC/EACTS guidelines for the management of valvular heart disease: The Task Force for the management of valvular heart disease of the European Society of Cardiology (ESC) and the European Association for Cardio- Thoracic Surgery (EACTS). Eur Heart J 2017;38:2739-91.

4. Forcillo J, Pellerin M, Perrault LP, et al. CarpentierEdwards pericardial valve in the aortic position: 25-years experience. Ann Thorac Surg 2013;96:486-93.

5. Bach DS, Kon ND. Long-term clinical outcomes 15 years after aortic valve replacement with the Freestyle stentless aortic bioprosthesis. Ann Thorac Surg 2014;97:544-51.

6. Capodanno D, Petronio AS, Prendergast B, et al. Standardized definitions of structural deterioration and valve failure in assessing long-term durability of transcatheter and surgical aortic bioprosthetic valves: a consensus statement from the European Association of Percutaneous Cardiovascular Interventions (EAPCI) endorsed by the European Society of Cardiology (ESC) and the European Association for Cardio-Thoracic Surgery (EACTS). Eur Heart J 2017;38:3382-90.

7. Dvir D, Bourguignon T, Otto CM, et al. Standardized Definition of Structural Valve Degeneration for Surgical and Transcatheter Bioprosthetic Aortic Valves. Circulation 
2018;137:388-99.

8. Salaun E, Clavel MA, Rodés-Cabau J, et al. Bioprosthetic aortic valve durability in the era of transcatheter aortic valve implantation. Heart 2018;104:1323-32.

9. Wyss TR, Bigler M, Stalder M, et al. Absence of Prosthesis-Patient Mismatch With the New Generation of Edwards Stented Aortic Bioprosthesis. Interact Cardiovasc Thorac Surg 2010;10:884-7.

10. Anselmi A, Ruggieri VG, Belhaj Soulami R, et al. Hemodynamic Results and Mid-term Follow-up of 850 19 to $23 \mathrm{~mm}$ Perimount Magna Ease Valves. Thorac Cardiovasc Surg 2019;67:274-81.

11. Devereux RB, Alonso DR, Luttas EM, et al. Echocardiographic assessment of left ventricular hypertrophy: comparison to necropsy findings. Am J Cardiol 1986;57:450.

12. Pibarot P, Dumesnil JG. Prosthesis-patient mismatch: definition, clinical impact, and prevention. Heart 2006;92:1022-9.

13. David TE, Armstrong S, Maganti M. Hancock II bioprosthesis for aortic valve replacement: the gold standard of bioprosthetic valves durability? Ann Thorac Surg 2010;90:775-81.

14. Yankah CA, Pasic M, Musci M, et al. Aortic valve replacement with the Mitroflow pericardial bioprosthesis: durability results up to 21 years. J Thorac Cardiovasc Surg 2008;136:688-96.

15. Bourguignon T, Bouquiaux-Stablo AL, Candolfi P, et

Cite this article as: Mayr B, Burri M, Vitanova K, Prinzing A, Goppel G, Krane M, Lange R, Günzinger R. Serial echocardiographic evaluation of the Perimount Magna Ease prosthesis. J Thorac Dis 2021;13(7):4104-4113. doi: 10.21037/jtd21-481 al. Very long-term outcomes of the Carpentier-Edwards Perimount valve in aortic position. Ann Thorac Surg 2015;99:831-7.

16. Bartuś K, Litwinowicz R, Kuśmierczyk M, et al. Primary safety and effectiveness feasibility study after surgical aortic valve replacement with a new generation bioprosthesis: one-year outcomes. Kardiol Pol 2018;76:618-24.

17. Bartus K, Litwinowicz R, Bilewska A, et al. Final 5-year outcomes following aortic valve replacement with a RESILIA $^{\mathrm{TM}}$ tissue bioprosthesis. Eur J Cardiothorac Surg 2021;59:434-41.

18. Yu W, Tam DY, Rocha RV, et al. Aortic Root Enlargement Is Safe and Reduces the Incidence of Patient-Prosthesis Mismatch: A Meta-analysis of Early and Late Outcomes. Can J Cardiol 2019;35:782-90.

19. Doenst T, Borger MA, Weisel RD, et al. Relation between aortic cross-clamp time and mortality: not as straightforward as expected. Eur J Cardiothorac Surg 2008;33:660-5.

20. Deutsch MA, Prinzing A, Fiegl K, et al. Early haemodynamic performance of a latest generation supra-annular aortic bioprosthesis: experience from a large single-centre series. Eur J Cardiothorac Surg 2016;49:1691-8.

21. Basra SS, Gopal A, Hebeler KR, et al. Clinical Leaflet Thrombosis in Transcatheter and Surgical Bioprosthetic Aortic Valves by Four-Dimensional Computed Tomography. Ann Thorac Surg 2018;106:1716-25. 